\title{
Design, Operational Experiences and Beam Results Obtained with the SNS H* Ion Source and LEBT at Berkeley Lab*
}

\author{
R. Keller and R. Thomae, E. O. Lawrence Berkeley National Laboratory \\ M. Stockli and R. Welton, Oak Ridge National Laboratory
}

\begin{abstract}
The ion source and Low-Energy Transport (LEBT) system that will provide $\mathrm{H}^{-}$ion beams to the Spallation Neutron Source (SNS)** Front End and the accelerator chain have been developed into a mature unit that fully satisfies the operational requirements through the commissioning and early operating phases of SNS. Compared to the early R\&D version, many features of the ion source have been improved, and reliable operation at $6 \%$ duty factor has been achieved producing beam currents in the 35-mA range and above. LEBT operation proved that the purely electrostatic focusing principle is well suited to inject the ion beam into the RFQ accelerator, including the steering and pre-chopping functions. This paper will discuss the latest design features of the ion source and LEBT, give performance data for the integrated system, and report on commissioning results obtained with the SNS RFQ and Medium-Energy Beam Transport (MEBT) system. Prospects for further improvements will be outlined in concluding remarks.
\end{abstract}

\section{INTRODUCTION}

Berkeley Lab has just completed building the linac injector (Front End, FE) for the Spallation Neutron Source project (SNS), and the commissioning of the entire system is proceeding. The main subsystems are the $\mathrm{H}^{-}$ion-source, the low-energy beam-transport system (LEBT), the $2.5-\mathrm{MeV}$ radio-frequency quadrupole (RFQ) accelerator, and the medium-energy beam-transport system (MEBT). Ion source and LEBT are the subject of this paper; their task is to create a $65-\mathrm{keV}, 38-\mathrm{mA}$ ion beam, to match and steer it into the RFQ, and to pre-chop it into mini-pulses of about $600 \mathrm{~ns}$ duration. The nominal duty factor is $6 \%$, with 1-ms macro-pulse length and $60-\mathrm{Hz}$ repetition rate.

Based upon the main design features of the SSC ion source, ${ }^{1}$ an R\&D version of the SNS ion source was built first to demonstrate the viability of the chosen approach, utilizing an rf driven discharge inside a multicusp plasma generator with magnetic filter, cesium enhancement, and electron suppression at low energy. ${ }^{2}$ This source version did not allow implementing cesium enhancement and electron suppression at the same time, but both features were proven to work satisfactorily in separate tests.

\footnotetext{
* This work is supported by the Director, Office of Science, Office of Basic Energy Sciences, of the U.S. Department of Energy under Contract No. DE-AC03-76SF00098.

** The SNS project is being carried out as a collaboration of six US Laboratories: Argonne National Laboratory (ANL), Brookhaven National Laboratory (BNL), Thomas Jefferson National Accelerator Facility (TJNAF), Los Alamos National Laboratory (LANL), E. O. Lawrence Berkeley National Laboratory (LBNL), and Oak Ridge National Laboratory (ORNL). SNS is managed by UT-Battelle, LLC, under contract DE-AC05-00OR22725 for the U.S. Department of Energy.
} 
For the LEBT, a purely electrostatic focusing system was chosen, thereby avoiding time-dependent space charge compensation usually encountered with magnetic LEBTs. The basic design was derived from a successful proton LEBT that had been used to inject beam into an RFQ accelerator on a test bed at LBNL. ${ }^{3}$

When the SNS project raised the performance goal for the Front End from 28 to $52 \mathrm{~mA}$, the design of a second-generation source had already progressed, and it appeared justified to carry on with the construction of this so-called startup source that aimed at a beam current of $35 \mathrm{~mA}$ to be delivered through the LEBT. The LEBT attached to this source had been modified from the original design to accommodate up to $70 \mathrm{~mA}$ of beam current, ${ }^{4}$ and the highest measured current value was $40 \mathrm{~mA} .^{5}$

The production version of the ion source and LEBT aims at generating and transporting a beam with 50-mA current, thought to be sufficient to satisfy the latest SNS design goal of $38 \mathrm{~mA}$ under the rather conservative assumption of $20 \%$ beam loss in the RFQ. As it turned out during the RFQ commissioning, ${ }^{6}$ this assumption might have been overly conservative because out of $36 \mathrm{~mA}$, maximum, beam injected into the RFQ up to $33 \mathrm{~mA}$ were passed through, and negligible losses are anticipated for the MEBT. It is also quite probable that the quality of the LEBT beam will improve somewhat as less than the nominal $50 \mathrm{~mA}$ have to be generated by the ion source. By now, three plasma generators and one LEBT, as shown in Fig. 1, have been built and tested, and the specific design features and performance data of this production system are discussed in the following sections.

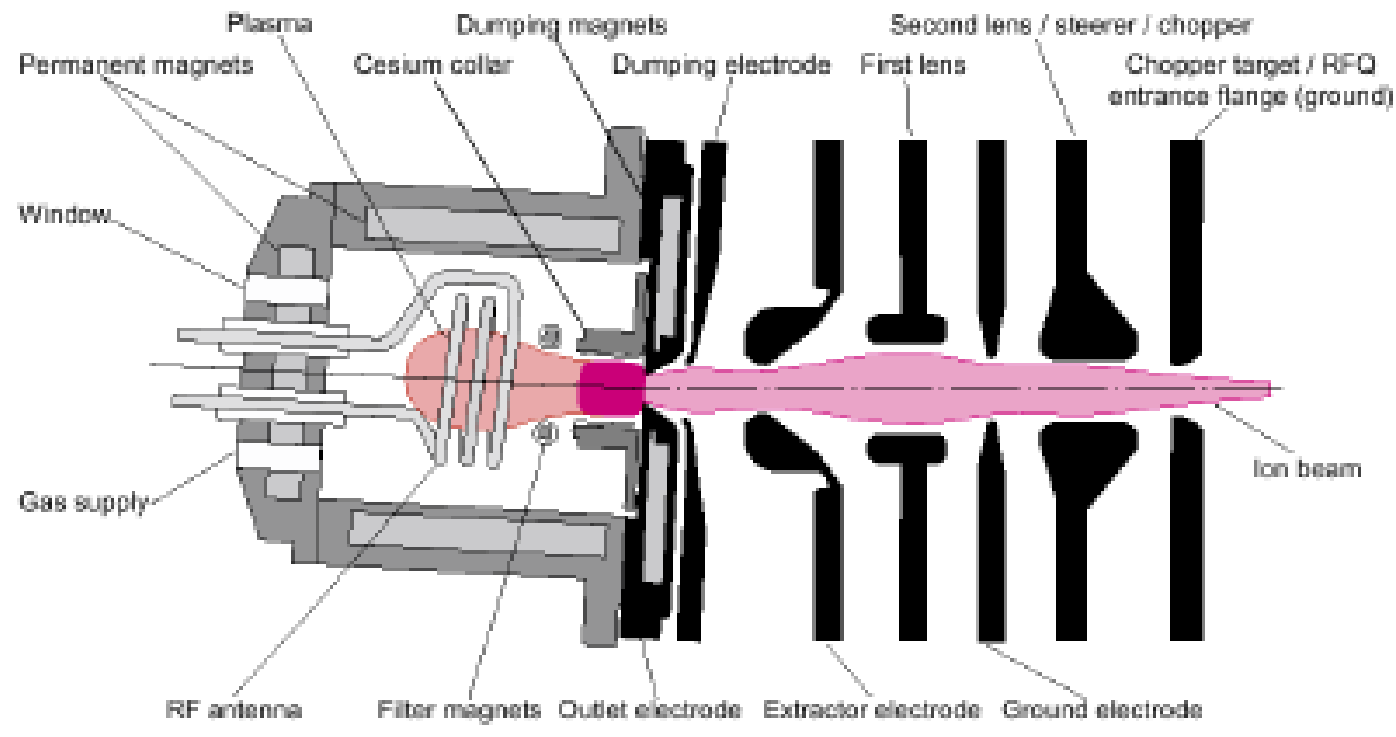

Figure 1. SNS Ion Source and LEBT layout, seen from the top. The actual orientation of filter and dumping magnet fields is orthogonal to the illustration plane. The width of the ion beam has been exaggerated in this figure to emphasize the focusing action of the two-lens LEBT. The second lens is split into four insulated quadrants to provide steering and chopping by applying separate pulsed and dc potentials. 


\section{ION SOURCE}

The production-version ion sources aim at generating $\mathrm{H}^{-}$beams of up to 50-mA current. Behind the LEBT, the transverse rms emittance goal for these beams is $0.2 \pi \mathrm{mm} \mathrm{mrad}$, normalized. For clarity, the term ion source in the context of this paper includes the plasma generator and electron dumping electrode, but no extractor which, instead, is considered part of the LEBT.

\section{Plasma Generation}

The design of the plasma generator is shown in Fig. 2. Tens of $\mathrm{kW}$ of $2-\mathrm{MHz}$ rf power are sustaining the hydrogen discharge inside the multi-cusp vessel.

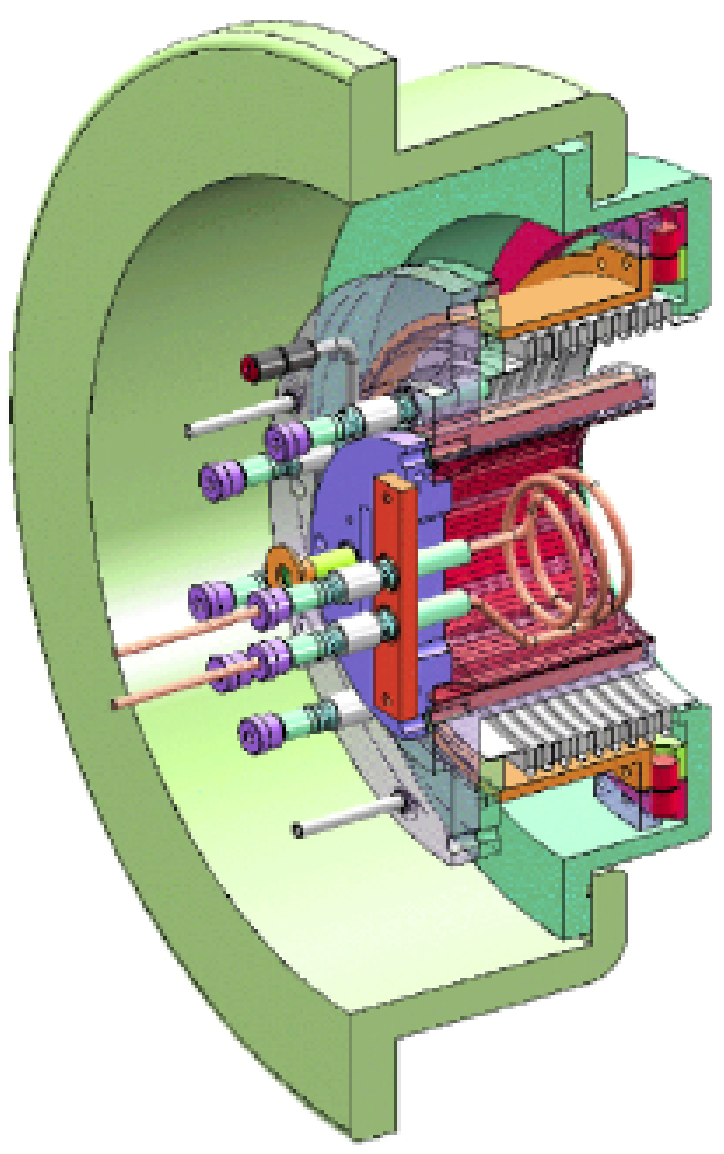

Figure 2. Multi-cusp plasma generator with rf antenna, inserted in the reentrant LEBT flange. The outlet plate and electron-dumping electrode are not shown here. The entire ion source can be pivoted horizontally around an axis in the outlet plane, on the right-hand side. 
The rf power is transmitted to the internal antenna through an inductive impedance-matching network. The antenna consists of a 2-1/2 winding copper coil, covered by a multi-layer porcelain coating. ${ }^{7}$ An antenna with $0.25-\mathrm{mm}$ coating underwent an endurance test at full duty factor, and the test was intentionally stopped after 107 hours and after verifying that the ion source delivered $20 \mathrm{~mA}$ of beam current at that time. An upgraded antenna with $0.8-\mathrm{mm}$ thick 10-layer coating produces the same plasma density and beam current for a given rf power level as the thin version (about $0.9 \mathrm{~mA}$ per $\mathrm{kW}$ of power) and is expected to last significantly longer.

A reliable method of plasma ignition at the beginning of every 1-ms pulse, was developed by maintaining a continuous, low-power discharge sustained by an additional $13.56-\mathrm{MHz}$ rf system. ${ }^{8}$ For reasons solely of practicality, we are using a capacitive impedance matcher for this continuous low-power rf. Initial difficulties were encountered with the 13.56-MHz amplifier switching off due to overload protection during the main pulses because of the impedance mismatch with the much denser plasma. This problem was eliminated by using an amplifier with higher nominal power rating than actually required to sustain the continuous low-density plasma alone.

During the development phase, we analyzed the light emitted from the plasma with a small spectroscope and were able to identify spectral emission lines of atomic hydrogen, cesium, and copper. ${ }^{8}$ This technique will allow characterizing the ion source plasma on-line and can be extended to predict the end of the antenna life while beam production is still ongoing.

\section{2. $H^{-}$Creation}

Negative hydrogen ions are preferentially created in the space confined by the cesium collar, the magnetic filter field, and the outlet electrode, see Fig. 1 . The filter field keeps energetic electrons that would destroy the $\mathrm{H}^{-}$ions away from the collar region. ${ }^{9}$ Volume production alone is sufficient to generate about $15 \mathrm{~mA}$ of beam current, but to reach the 50-mA level, cesium enhancement is needed. For that purpose, the collar is fitted with eight cesium-chromate containers and is thermally isolated from the source body to allow it heating up to several hundred degrees $C$. The presence of about $1 / 2$ a mono-layer of cesium inner collar surface not only multiplies the abundance of negative ions in the discharge plasma by about a factor of three, but it also reduces the abundance of electrons in the extracted beam by one order of magnitude. Details of the enhancing cesium action are given elsewhere. ${ }^{10}$

To best utilize the cesium, a freshly cleaned plasma generator is operated at full duty factor for about $15 \mathrm{~min}$., heating the cesium collar to more than $500^{\circ} \mathrm{C}$ by forcing hot air through the collar wall and utilizing the rf power as a source of heat. After this initial conditioning, the collar is cooled down by roomtemperature air and kept at about $280^{\circ} \mathrm{C}$ for optimal beam production. The cesium layer can then last for several days. Additional cesium reconditioning can be performed in-situ as needed. After controlled venting to atmospheric pressure, using dry nitrogen first, in order, for example, to change out a defective rf antenna, the source can be restarted without any cleaning because the amount of deposited cesium is very small. 
Recently a new collar has been developed that seamlessly merges with the outlet aperture. This design brings three major advantages: 1), it provides for keeping the surfaces around the outlet aperture at the same temperature as the collar; 2), with the help of an isolating centering ring, it allows precise aligning of the collar to the axis of the outlet aperture and at the same time biasing the entire unit to an optimal potential with respect to the source body; ${ }^{11}$ and 3 ), it allows modifying the contour of the outlet aperture as will be discussed below, without having to build another main outlet flange. This integrated collar/outlet aperture has been fabricated but so far not yet been tested in the SNS ion source. Its design is shown in Fig. 3.

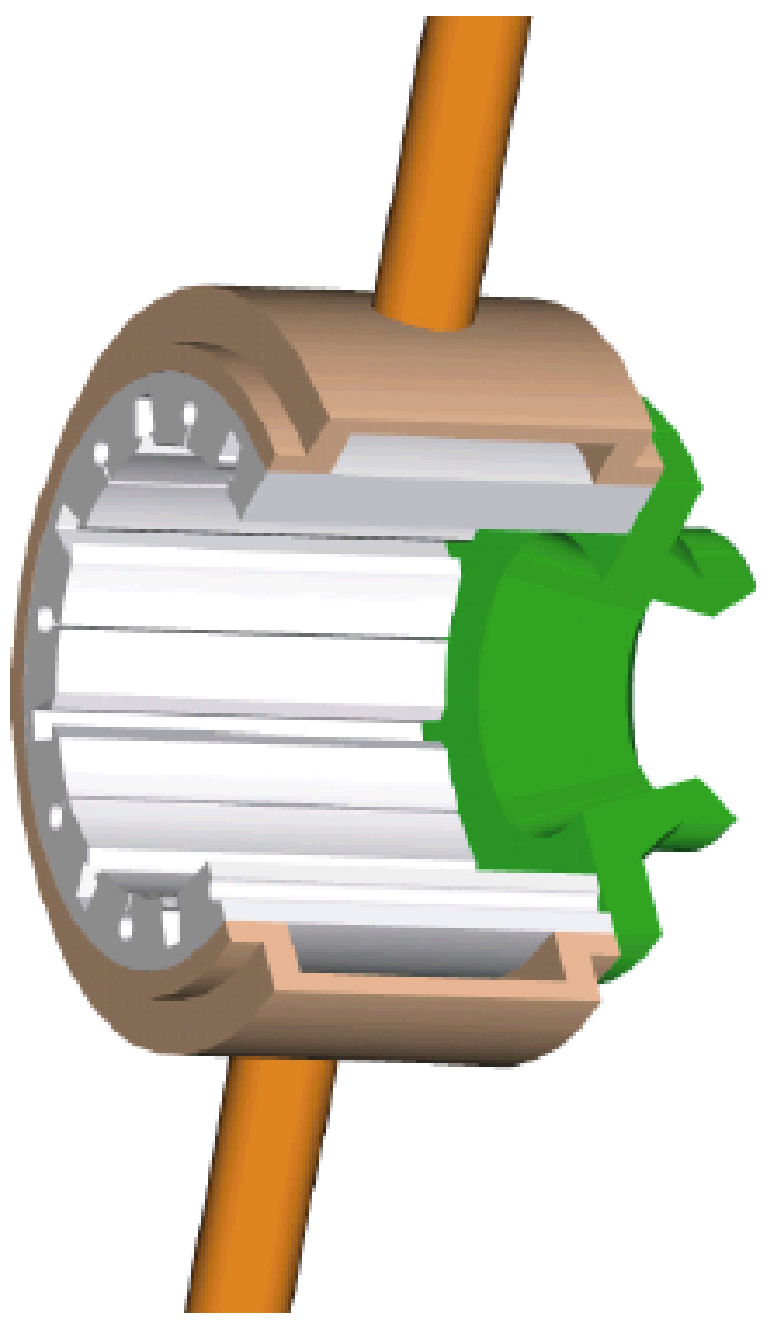

Figure 3. Integrated cesium collar with slots for cesium containers (left) and outlet aperture (right). Either heated or room-temperature air is conducted from the lower to the upper tube, passing around the collar, to keep it at optimal temperatures for initial cesiation or beam production. 


\section{LOW-ENERGY BEAM TRANSPORT}

\section{Electron Dumping}

When a negative-ion beam is being extracted from a plasma, a substantial amount of electrons is extracted as well. These electrons create two kinds of problems: the first due to their space charge, especially near the outlet aperture where the particle velocities are low; and the second one due to the power load to the structure where the electron beam is deposited. Unfortunately, trying to solve the second of these problems by separating the electrons at low energy from the ions increases the severity of the first problem. The compromise that was sought with the design of the SNS ion source consists in depositing the electrons on a dedicated 'dumping electrode' at moderate energies of about $5 \mathrm{keV}$ as compared to the full ion-beam energy of $65 \mathrm{keV}$. Mechanically, this dumping electrode can be considered to be a part of the ion source proper because it is supported by and insulated against the outlet electrode. A spark gap ensures that no excessive voltage can be built up between these two electrodes when the main extraction voltage breaks down.

The separation process is accomplished by a set of permanent magnets inserted inside the outlet electrode in a so-called Halbach configuration; ${ }^{12}$ the magnets create a sharply peaked dipole field in the extraction gap. Because of the uncertainty of the actual plasma meniscus location with respect to the dumping field, aggravated by the fact that this location might well depend on the plasma density inside the collar volume as well as on the degree of cesiation, the actual electron trajectories are not well enough known under all conditions, and in most cases some fraction of the electrons misses the dumping electrode entirely. These electrons are accelerated to full beam energy and hit either the extractor electrode or its support structure. A water-cooled shield has now been installed on the support structure to take care of the heat load. It is expected that the new, integrated collar/outlet electrode described above will definitively resolve this problem.

Because of the steering action of the dumping magnetic field, not only on the electrons, but also on the ions, the entire ion source is tilted by an adjustable angle of about $3^{\circ}$ with respect to the LEBT axis.

\section{Beam Formation}

When the SNS Ion Source and LEBT were developed, only positive-ion codes were available to us for simulation work. The code IGUN ${ }^{13}$ had proven to be very reliable in predicting trajectories of positive ions, and we used it by implicitly assuming the negative ions to be protons and adding a fixed amount of current to the expected ion beam current to represent the space charge of the electrons in the zone between outlet and dumping electrodes. ${ }^{4}$ Even though this method was good enough to get a very useful LEBT design, these simulations proved to be inadequate for the purpose of optimizing the extraction system details. In the meantime, the code PBGUNS ${ }^{14}$ had been extended by its author to properly address negative-ion extraction problems, and with its help an improved outlet- 
electrode design was created that was implemented in the new, integrated collar/outlet electrode.

The main difference between IGUN and PBGUNS results is their prediction where the plasma meniscus would be anchored on the outlet aperture, as illustrated in Fig. 4. Experimental results discussed below support the validity of the PBGUNS results. The non-uniformly curved plasma meniscus as predicted by these latter simulations for the original SNS ion-source outlet geometry produces significantly distorted emittances.

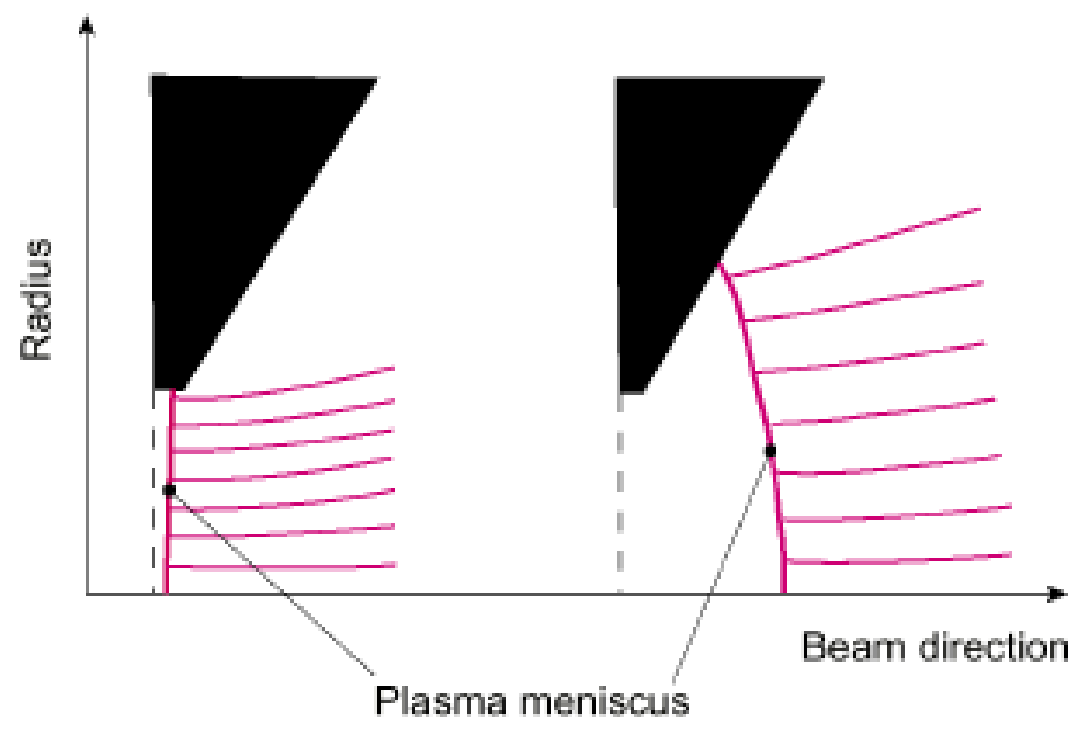
a) IGUN
b) PBGUNS

Figure 4. Schematic simulation results of the beam formation process with the original SNS ion-source outlet geometry. In close agreement with actual emittance measurements, the PBGUNS simulations (b), suggest that the plasma meniscus is not anchored at the edge of the outlet aperture but protrudes significantly beyond the ideal outlet plane. This effect leads to a larger size of the meniscus than was predicted for protons by IGUN and to a non-uniform meniscus curvature, both resulting in larger and more distorted emittances.

A systematic study of outlet-aperture contours ${ }^{15}$ indicated that a longer channel such as the one shown in Fig. 3, as opposed to the knife-edge shape seen in Fig. 4, would lead to a smaller and essentially flat meniscus, resulting in significantly smaller emittance size.

\section{Principal LEBT Functions}

Apart from the beam formation, the main purpose of the LEBT is to transport the beam to the RFQ and give it Twiss parameter values matching the injection 
requirements. Efficient pumping of the gas load produced by the plasma generator is another task that was addressed by giving the electrode support structures highly transparent shapes, as shown in Fig. 5.

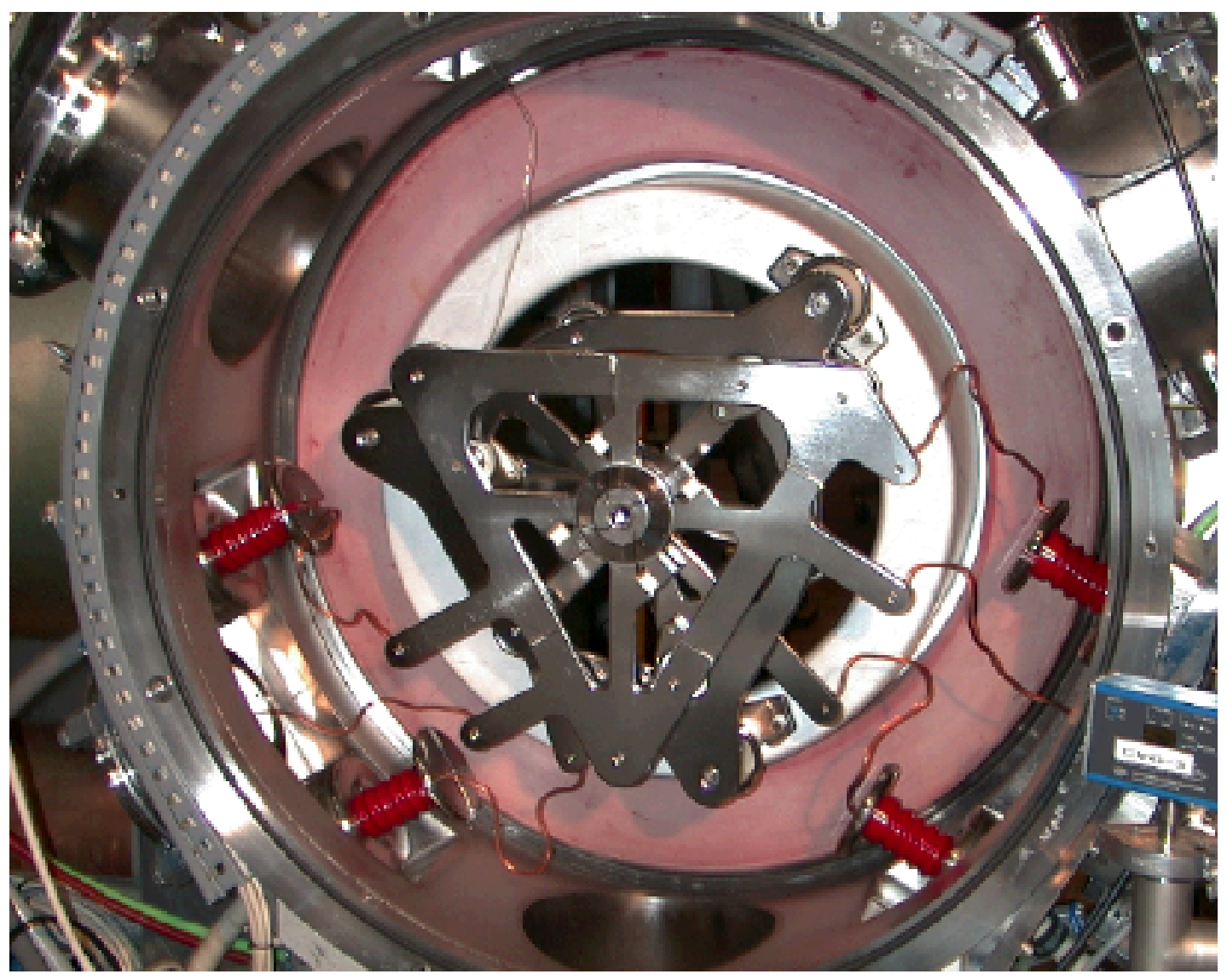

Figure 5. View of the LEBT electrodes inside the vacuum tank, from the downstream end. The first electrode seen is the center electrode of the second lens, split into four isolated quadrants.

The focusing action of the electrostatic two-lens system, captured in a tuning matrix, works as predicted by simulations, but generation of less than nominal beam current results in a narrower beam size inside the first lens and effectively attenuates its focusing power. For that reason, the extraction gap was widened by $4 \mathrm{~mm}$ as compared to the nominal size of $20 \mathrm{~mm}$ to handle beams in the 35$\mathrm{mA}$ range during the RFQ commissioning. The widened gap allows the beam to expand more and thus enables the first lens to carry its share of the focusing action.

The functions of pre-chopping and static steering are implemented by applying pulsed voltage signals and, independently, dc potentials to the four quad- 
rants of the center electrode of the second lens. Chopping voltages of $\pm 2.5 \mathrm{kV}$ amplitude are applied to adjacent pairs of quadrants such as to deflect the beam alternatingly into the four diagonal directions and thus distribute the local heat load on the ring-shaped chopper target that is imbedded in the RFQ entrance flange. Not all of the chopped beam is intercepted by this ring target; the remaining particles are deposited inside the RFQ cavities, well away from the vane tips that are oriented in the horizontal and vertical directions.

\section{BEAM RESULTS}

The nominal beam-current goal of $50 \mathrm{~mA}$ pulse average measured at full $6 \%$ duty factor downstream of the LEBT was reached about a year ago, still with the nominal gap width installed. The Faraday cup used for all these measurements is magnetically isolated and electrically biased to suppress secondary electrons as well as electrons that might have been transported together with the negative ions. The peak current at the beginning of every pulse even reached $68 \mathrm{~mA}$, as shown in Fig. 6. The pulse shape had not been optimized for uniformity, and if the initial peak cannot be suppressed by just applying suitable discharge conditions the option of shaping the 2-MHz rf-power waveform is available. With the extraction gap between dumping electrode and LEBT increased by $4 \mathrm{~mm}$ for RFQ commissioning, as discussed above, up to $36 \mathrm{~mA}$ were measured, and the pulse shape was much more uniform.

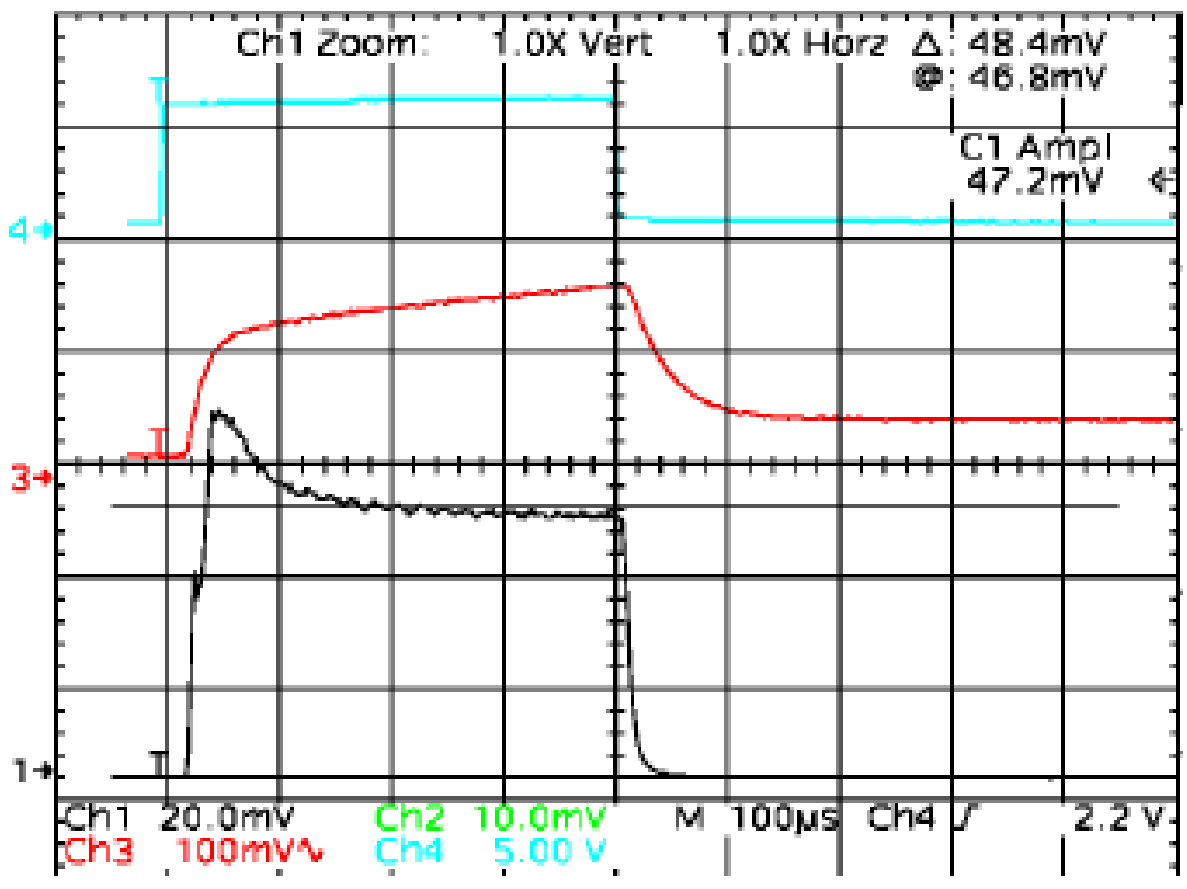

Figure 6. Beam pulses (50 mA average, $68 \mathrm{~mA}$ peak) measured downstream of the LEBT with the nominal extraction gap. 
The horizontal and vertical emittances of a focused 33-mA LEBT beam are shown in Fig. 7. They were measured using an Allison-type emittance device with electrostatic angle scan. ${ }^{16}$ Pronounced distortions are evident slightly beyond the $10 \%$ intensity level, but after subtracting background signals from the raw data , the rms sizes are very close to or even better than the nominal values.

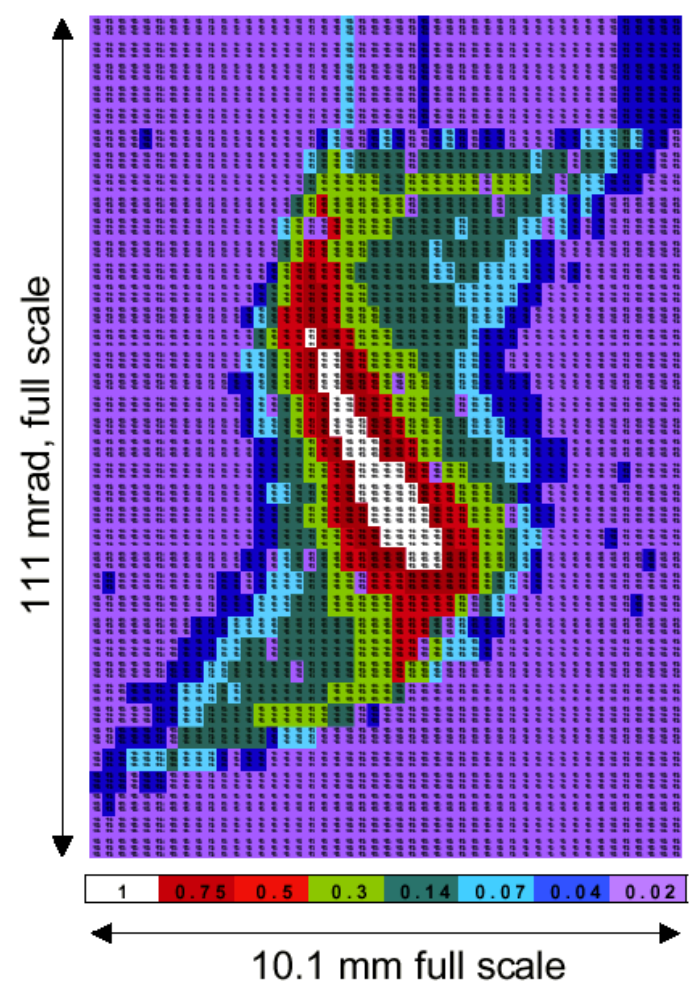

Horizontal, 0.22 pi mm mrad

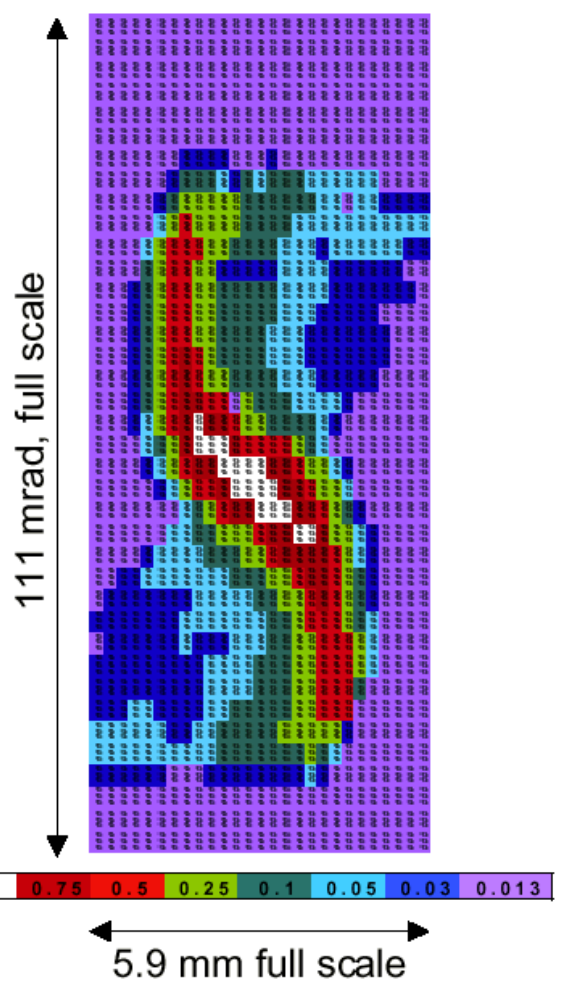

Vertical, 0.15 pi $\mathrm{mm}$ mrad

Figure 7. Horizontal and vertical LEBT emittances for a 33-mA beam out of the LEBT, after subtraction of background signals: $2.1 \%$ of peak intensity horizontal and $1.3 \%$ vertical, respectively.

A round-the-clock endurance test of the ion source and LEBT was conducted over more than a week, continuously producing beam with about $25 \mathrm{~mA}$ current at $3 \%$ duty factor. This test led to the elimination of several minor technical flaws and was very successful overall. The sparking rate steadily improved to about once per hour, and operations were interrupted only a few times, mostly because of external events that affected the ion source operation. The test proved that the beam-generating system is ready to support commissioning of the SNS accelerators and even SNS operations for the first few years, and in addition it helped dealing with a few remaining design weaknesses. 
In fact, the commissioning of the SNS RFQ at low duty factor ${ }^{6}$ was aided by very stable performance of Ion Source and LEBT. Emittances of diverging beams measured $450 \mathrm{~mm}$ downstream of the RFQ do not show any signs of residual distortions; apparently the RFQ clips the most aberrant parts of the injected phase-space distributions while still allowing more than $90 \%$ of the beam to pass through.

The functionality of the LEBT pre-chopper system has been tested as well, and rise and fall times of $25 \mathrm{~ns}$ were measured, beating the nominal requirement by a factor of 2 and reducing the thermal load on the MEBT chopper-target in proportion. The beam signals were not clear enough to allow a precise determination of the pre-chopper attenuation factor, but a value around $1 \times 10^{-3}$ appears quite plausible from extrapolations of results obtained at less than nominal chopping voltages.

\section{OUTLOOK}

The Ion Source and LEBT systems built for the SNS Linac injector have come a long way towards fulfilling their nominal performance goals in terms of beam current and quality as well as overall functionality. The RFQ commissioning results even appear to allow for some margin in terms of the rms emittance sizes. It remains to be seen, however, how much the noted aberration tails of the LEBT beam are going to contribute to halo generation in the MEBT and even the Linac sections farther downstream. The rms emittance-concept evidently is too coarse a measure to ascertain such effects. Even though the SNS project is going to provide for halo scrapers in the MEBT a reduction of the emittance distortions after the LEBT appears to be highly desirable, and the beam formation system offers itself for the first steps in that direction.

Quite some hope rests on the expected performance of the integrated collar/ outlet unit with improved outlet contour, but if this turned out to be insufficient one could go further along this path by implementing an electron dumping system at intermediate energies around $15 \mathrm{keV}$ as used already elsewhere. ${ }^{17}$ The advantage of such an approach lies in the fact that the parasitic electrons are at first being extracted in a geometry with cylindrical symmetry, avoiding the build-up of asymmetric space-charge forces that could act on the very slow ions near the meniscus. When the electrons are finally being separated from the ion beam their own speed is high enough that asymmetric space-charge forces become essentially negligible. The price for utilizing such a dumping system is to be paid in having to remove a higher thermal load from the catcher electrode, and possibly in a higher rate of x-ray emission.

It is debatable if one should work towards the elimination of the epoxy-based joints in the present LEBT assembly. Of course, overheating of these structures could lead to ungluing and downtime to execute the necessary repairs, but uncontrolled overheating of the LEBT electrodes for lack of a 'mechanical fuse' could lead to even more serious deformation of these parts and even worse loss of time. Installing water-cooling lines as already initiated with the present extractor support appears to be the better remedy in the long run, in addition to carefully avoiding unwanted heat deposition in the first place. 
The actual lifetime of an ion source is a never-ending topic for accelerator projects, and for the SNS Ion Source, the rf antenna appears to be the most critical element in this respect. Very significant progress has been made with the antenna development reported in this paper, but the ultimate lifetime of the currently employed antennas under nominal operation conditions is simply not yet known. While this issue might be relevant for SNS only several years from now, when the actual beam-production phase of the facility has started, it certainly would be good to know much earlier that the nominal time of three weeks between scheduled services can be reached at will.

To solidly achieve the stated goals, a new approach to the discharge technology might well be required, and two options are currently being considered among the SNS collaboration. They involve either use of an external antenna such as the one that gave very respectable results in low duty-factor operation, ${ }^{18}$ or, as a more fundamental change, a pulsed-dc discharge mode supported by a plasma cathode.

\section{ACKNOWLEDGEMENTS}

The authors would like to acknowledge the support by a large number of SNS staff at LBNL and ORNL, not necessarily listed in the references, who supported this work; thanks are due in particular to R. Gough, R. Yourd, R. DiGennaro, A. Ratti, S. Lewis, T. Schenkel, D. Cheng, K. N. Leung, J. Greer, J. W. Staples, D. Syversrud, W. Abraham, T. Kuneli, N. Ybarrolaza, C. Lionberger, P. Cull, M. Hoff, J. Pruyn, R. MacGill, M. Monroy, M. Regis, J. Dougherty, D. Garfield, and K. Barat.

\section{REFERENCES}

1. K. Saadatmand, G. Arbique, J. Hebert, R. Valicenti, and K. N. Leung, "Performance of a High Current $\mathrm{H}^{-}$Radio Frequency Volume Ion source," Rev. Sci. Instr. 67 (3), p. 1318-1320 (1996).

2. M.A. Leitner, D.W. Cheng, S.K. Mukherjee, J.Greer, P.K. Scott, M.D. Williams, K.N. Leung, R. Keller, R.A. Gough, "High-Current, High-Duty-Factor Experiments with the RF Driven H- Ion Source for the Spallation Neutron Source," PAC '99, New York (1999).

3. J. W. Staples, M. D. Hoff, and C. F. Chan, "All-electrostatic Split LEBT Test Results," Linac '96, (1996).

4. R J. Reijonen, R. Thomae, and R. Keller, "Evolution of the LEBT Layout for SNS," Paper MOD19,Linac 2000, Monterey (2000).

5. R. Thomae, P. Bach, R. Gough, J. Greer, R. Keller, and K. N. Leung, "Measurements on the H - Ion Source and Low Energy Beam Transport Section for the SNS Front-End Systems," Paper MOD09, Linac 2000, Monterey (2000).

6. R. Keller for the SNS Front-End Systems team, "Commissioning of the SNS Front-End Systems at Berkeley Lab," submitted to EPAC '02, Paris (2002). 
7. R. F. Welton, M.P. Stockli, Y. Kang, M. Janney, R. Keller, T. Schenkel, R. Thomae, and S. Shukla, "Ion Source Antenna Development for the Spallation Neutron Source," Rev. Sci. Instrum. 73 (2), p. 1008 - 1012 (2002).

8. T. Schenkel, J. W. Staples, R. W. Thomae, J. Reijonen, R. A. Gough, K. N. Leung, R. Keller, R. Welton, and M. Stockli, "Plasma Ignition schemes for the Spallation Neutron Source Radio-Frequency Driven $\mathrm{H}^{-}$Source," Rev. Sci. Instrum. 73 (2), p. 1017 - 1019 (2002).

9. K.-N. Leung in I. G. Brown, ed., "The Physics and Technology of Ion Sources," ch. 17, "Negative Ion Sources," p. 355, John Wiley \& Sons, New York (1989).

10. R. Welton, M. Stockli, R. Keller, and R. Thomae, "Enhancing Surface Ionization and Beam Formation in Volume-type H- Ion Sources," submitted to EPAC '02, Paris (2002).

11. J. Peters, "The Plasma-Vacuum Transition in RF Sources for Negative Hydrogen Ions," Rev. Sci. Instrum. 73 (2), p. 900 - 902 (2002).

12. K. Halbach, Nucl. Instrum. Methods 169, p.1 (1980).

13. R. Becker, "New Features in the Simulation of Ion Extraction with IGUN," Proceedings of EPAC '98, Stockholm (1998).

14. J.E. Boers, PBGUNS Manual, available through Thunderbird Simulations, Garland, TX, 75042.

15. R. F. Welton, M.P. Stockli, J.E. Boers, R. Rauniyar, R. Keller, J.W. Staples, and R.W. Thomae, "Simulation of the Ion Source Extraction and Low-Energy Beam Transport Systems for the Spallation Neutron Source," Rev. Sci. Instrum. 73 (2), p. 1013 - 1016 (2002).

16. P. W. Allison, J. D. Sherman, and D. B. Holtkamp, IEEE Trans. Nucl. Sci. NS30, 2204 (1983).

17. H. Oguri, Y. Okumura, and K. Hasegawa, "Development of an H- Ion Source for the High Intensity Proton Linac," Rev. Sci. Instrum. 73 (2), p. 1021 - 1023 (2002).

18. J. Peters, "Internal versus External RF Coupling into a Volume Source," submitted to EPAC '02, Paris (2002). 